\title{
MULTIPLE NONNEGATIVE SOLUTIONS FOR BVPs OF FOURTH-ORDER DIFFERENCE EQUATIONS
}

\author{
JIAN-PING SUN
}

Received 31 March 2006; Revised 5 September 2006; Accepted 18 September 2006

First, existence criteria for at least three nonnegative solutions to the following boundary value problem of fourth-order difference equation $\Delta^{4} x(t-2)=a(t) f(x(t)), t \in[2, T]$, $x(0)=x(T+2)=0, \Delta^{2} x(0)=\Delta^{2} x(T)=0$ are established by using the well-known LeggettWilliams fixed point theorem, and then, for arbitrary positive integer $m$, existence results for at least $2 m-1$ nonnegative solutions are obtained.

Copyright (c) 2006 Jian-Ping Sun. This is an open access article distributed under the Creative Commons Attribution License, which permits unrestricted use, distribution, and reproduction in any medium, provided the original work is properly cited.

\section{Introduction}

Recently, boundary value problems (BVPs) of difference equations have received considerable attention from many authors, see $[1-5,7-9,12-19]$ and the references therein. In particular, Zhang et al. [19] established the existence of positive solution to the fourthorder BVP

$$
\begin{gathered}
\Delta^{4} x(t-2)=\lambda a(t) f(t, x(t)), \quad t \in N, 2 \leq t \leq T, \\
x(0)=x(T+2)=0, \\
\Delta^{2} x(0)=\Delta^{2} x(T)=0
\end{gathered}
$$

by using the method of upper and lower solutions, and then Sun [15] obtained the existence of one positive solution for the following fourth-order BVP:

$$
\begin{gathered}
\Delta^{4} x(t-2)=a(t) f(x(t)), \quad t \in[2, T], \\
x(0)=x(T+2)=0 \\
\Delta^{2} x(0)=\Delta^{2} x(T)=0
\end{gathered}
$$




\section{Solutions to BVPs of fourth-order difference equations}

under the assumption that $f$ is either superlinear or sublinear, where $T>2$ is a fixed positive integer, $\Delta^{m}$ denotes the $m$ th forward difference operator with stepsize 1 , and $[a, b]=\{a, a+1, \ldots, b-1, b\} \subset \mathbb{Z}$ the set of all integers. Our main tool was the GuoKrasnosel'skii fixed point theorem in cone $[6,10]$.

In this paper we will continue to consider the BVP (1.2). First, existence criteria for at least three nonnegative solutions to the BVP (1.2) are established by using the wellknown Leggett-Williams fixed point theorem [11], and then, for arbitrary positive integer $m$, existence results for at least $2 m-1$ nonnegative solutions to the BVP (1.2) are obtained.

Throughout this paper, we assume that the following two conditions are satisfied.

(C1) $f:[0, \infty) \rightarrow[0, \infty)$ is continuous.

(C2) $a:[2, T] \rightarrow[0, \infty)$ is not identical zero.

In order to obtain our main results, we need the following concepts and LeggettWilliams fixed point theorem.

Let $E$ be a real Banach space with cone $P$. A map $\alpha: P \rightarrow[0,+\infty)$ is said to be a nonnegative continuous concave functional on $P$ if $\alpha$ is continuous and

$$
\alpha(t x+(1-t) y) \geq t \alpha(x)+(1-t) \alpha(y)
$$

for all $x, y \in P$ and $t \in[0,1]$. Let $a, b$ be two numbers such that $0<a<b$ and let $\alpha$ be a nonnegative continuous concave functional on $P$. We define the following convex sets:

$$
\begin{gathered}
P_{a}=\{x \in P:\|x\|<a\}, \\
P(\alpha, a, b)=\{x \in P: a \leq \alpha(x),\|x\| \leq b\} .
\end{gathered}
$$

Theorem 1.1 (Leggett-Williams fixed point theorem). Let $A: \overline{P_{c}} \rightarrow \overline{P_{c}}$ be completely continuous and let $\alpha$ be a nonnegative continuous concave functional on $P$ such that $\alpha(x) \leq\|x\|$ for all $x \in \overline{P_{c}}$. Suppose there exist $0<d<a<b \leq c$ such that

(i) $\{x \in P(\alpha, a, b): \alpha(x)>a\} \neq \phi$ and $\alpha(A x)>a$ for $x \in P(\alpha, a, b)$;

(ii) $\|A x\|<d$ for $\|x\| \leq d$;

(iii) $\alpha(A x)>a$ for $x \in P(\alpha, a, c)$ with $\|A x\|>b$.

Then $A$ has at least three fixed points $x_{1}, x_{2}, x_{3}$ in $\overline{P_{c}}$ satisfying

$$
\left\|x_{1}\right\|<d, \quad a<\alpha\left(x_{2}\right), \quad\left\|x_{3}\right\|>d, \quad \alpha\left(x_{3}\right)<a .
$$

\section{Main results}

For convenience, we denote

$$
\begin{aligned}
& G_{1}(t, s)=\frac{1}{T} \begin{cases}(t-1)(T+1-s), & 1 \leq t \leq s \leq T, \\
(s-1)(T+1-t), & 2 \leq s \leq t \leq T+1,\end{cases} \\
& G_{2}(t, s)=\frac{1}{T+2} \begin{cases}t(T+2-s), & 0 \leq t \leq s \leq T+1, \\
s(T+2-t), & 1 \leq s \leq t \leq T+2,\end{cases}
\end{aligned}
$$




$$
\begin{aligned}
& D=\max _{t \in[0, T+2]} \sum_{s=1}^{T+1} G_{2}(t, s) \sum_{v=2}^{T} G_{1}(s, v) a(v), \\
& C=\min _{t \in[2, T]} \sum_{s=1}^{T+1} G_{2}(t, s) \sum_{v=2}^{T} G_{1}(s, v) a(v) .
\end{aligned}
$$

It is easily seen from the expression of $G_{2}(t, s)$ that

$$
\begin{gathered}
G_{2}(t, s) \leq G_{2}(s, s), \quad(t, s) \in[0, T+2] \times[1, T+1], \\
G_{2}(t, s) \geq \frac{1}{T+1} G_{2}(s, s), \quad(t, s) \in[1, T+1] \times[1, T+1] .
\end{gathered}
$$

Our main result is the following theorem.

Theorem 2.1. Assume that there exist numbers $d$, $a$, and $c$ with $0<d<a<(T+1) a<c$ such that

$$
\begin{gathered}
f(x)<\frac{d}{D}, \quad x \in[0, d], \\
f(x)>\frac{a}{C}, \quad x \in[a,(T+1) a], \\
f(x)<\frac{c}{D}, \quad x \in[0, c] .
\end{gathered}
$$

Then the BVP (1.2) has at least three nonnegative solutions.

Proof. Let the Banach space $E=\{x:[0, T+2] \rightarrow R\}$ be equipped with the norm

$$
\|x\|=\max _{t \in[0, T+2]}|x(t)| .
$$

We define

$$
P=\{x \in E: x(t) \geq 0, t \in[0, T+2]\},
$$

then it is obvious that $P$ is a cone in $E$.

For $x \in P$, we define

$$
\begin{gathered}
\alpha(x)=\min _{t \in[2, T]} x(t) \\
(A x)(t)=\sum_{s=1}^{T+1} G_{2}(t, s) \sum_{v=2}^{T} G_{1}(s, v) a(v) f(x(v)), \quad t \in[0, T+2] .
\end{gathered}
$$

It is easy to check that $\alpha$ is a nonnegative continuous concave functional on $P$ with $\alpha(x) \leq$ $\|x\|$ for $x \in P$ and that $A: P \rightarrow P$ is completely continuous and fixed points of $A$ are solutions of the BVP (1.2).

We first assert that if there exists a positive number $r$ such that $f(x)<r / D$ for $x \in[0, r]$, then $A: \overline{P_{r}} \rightarrow P_{r}$. 
4 Solutions to BVPs of fourth-order difference equations

Indeed, if $x \in \overline{P_{r}}$, then for $t \in[0, T+2]$,

$$
\begin{aligned}
(A x)(t) & =\sum_{s=1}^{T+1} G_{2}(t, s) \sum_{v=2}^{T} G_{1}(s, v) a(v) f(x(v)) \\
& <\frac{r}{D} \sum_{s=1}^{T+1} G_{2}(t, s) \sum_{v=2}^{T} G_{1}(s, v) a(v) \\
& \leq \frac{r}{D} \max _{t \in[0, T+2]} \sum_{s=1}^{T+1} G_{2}(t, s) \sum_{v=2}^{T} G_{1}(s, v) a(v)=r .
\end{aligned}
$$

Thus, $\|A x\|<r$, that is, $A x \in P_{r}$.

Hence, we have shown that if (2.3) and (2.5) hold, then $A$ maps $\bar{P}_{d}$ into $P_{d}$ and $\bar{P}_{c}$ into $P_{c}$.

Next, we assert that $\{x \in P(\alpha, a,(T+1) a): \alpha(x)>a\} \neq \phi$ and $\alpha(A x)>a$ for all $x \in$ $P(\alpha, a,(T+1) a)$.

In fact, the constant function

$$
\frac{(T+2) a}{2} \in\{x \in P(\alpha, a,(T+1) a): \alpha(x)>a\} .
$$

Moreover, for $x \in P(\alpha, a,(T+1) a)$, we have

$$
(T+1) a \geq\|x\| \geq x(t) \geq \min _{t \in[2, T]} x(t)=\alpha(x) \geq a
$$

for all $t \in[2, T]$. Thus, in view of (2.4), we see that

$$
\begin{aligned}
\alpha(A x) & =\min _{t \in[2, T]} \sum_{s=1}^{T+1} G_{2}(t, s) \sum_{v=2}^{T} G_{1}(s, v) a(v) f(x(v)) \\
& >\frac{a}{C} \min _{t \in[2, T]} \sum_{s=1}^{T+1} G_{2}(t, s) \sum_{v=2}^{T} G_{1}(s, v) a(v)=a
\end{aligned}
$$

as required.

Finally, we assert that if $x \in P(\alpha, a, c)$ and $\|A x\|>(T+1) a$, then $\alpha(A x)>a$.

To see this, suppose $x \in P(\alpha, a, c)$ and $\|A x\|>(T+1) a$, then in view of (2.2), we have

$$
\begin{aligned}
\alpha(A x) & =\min _{t \in[2, T]} \sum_{s=1}^{T+1} G_{2}(t, s) \sum_{v=2}^{T} G_{1}(s, v) a(v) f(x(v)) \\
& \geq \frac{1}{T+1} \sum_{s=1}^{T+1} G_{2}(s, s) \sum_{v=2}^{T} G_{1}(s, v) a(v) f(x(v)) \\
& \geq \frac{1}{T+1} \sum_{s=1}^{T+1} G_{2}(t, s) \sum_{v=2}^{T} G_{1}(s, v) a(v) f(x(v))
\end{aligned}
$$


for $t \in[0, T+2]$. Thus

$$
\begin{aligned}
\alpha(A x) & \geq \frac{1}{T+1} \max _{t \in[0, T+2]} \sum_{s=1}^{T+1} G_{2}(t, s) \sum_{v=2}^{T} G_{1}(s, v) a(v) f(x(v)) \\
& =\frac{1}{T+1}\|A x\|>\frac{1}{T+1}(T+1) a=a .
\end{aligned}
$$

To sum up, all the hypotheses of the Leggett-Williams theorem are satisfied. Hence $A$ has at least three fixed points, that is, the BVP (1.2) has at least three nonnegative solutions $u, v$, and $w$ such that

$$
\begin{gathered}
\|u\|<d, \quad a<\min _{t \in[2, T]} v(t), \quad\|w\|>d, \\
\min _{t \in[2, T]} w(t)<a .
\end{gathered}
$$

The proof is complete.

Corollary 2.2. Let $m$ be an arbitrary positive integer. Assume that there exist numbers $d_{j}$ $(1 \leq j \leq m)$ and $a_{h}(1 \leq h \leq m-1)$ with $0<d_{1}<a_{1}<(T+1) a_{1}<d_{2}<a_{2}<(T+1) a_{2}<$ $\cdots<d_{m-1}<a_{m-1}<(T+1) a_{m-1}<d_{m}$ such that

$$
\begin{gathered}
f(x)<\frac{d_{j}}{D}, \quad x \in\left[0, d_{j}\right], 1 \leq j \leq m, \\
f(x)>\frac{a_{h}}{C}, \quad x \in\left[a_{h},(T+1) a_{h}\right], 1 \leq h \leq m-1 .
\end{gathered}
$$

Then, the BVP (1.2) has at least $2 m-1$ nonnegative solutions in $\overline{P_{d_{m}}}$.

Proof. We prove this conclusion by induction.

First, for $m=1$, we know from (2.16) that $A: \overline{P_{d_{1}}} \rightarrow P_{d_{1}} \subset \overline{P_{d_{1}}}$, then, it follows from Schauder fixed point theorem that the BVP (1.2) has at least one nonnegative solution in $\overline{P_{d_{1}}}$.

Next, we assume that this conclusion holds for $m=k$. In order to prove that this conclusion also holds for $m=k+1$, we suppose that there exist numbers $d_{j}(1 \leq j \leq k+1)$ and $a_{h}(1 \leq h \leq k)$ with $0<d_{1}<a_{1}<(T+1) a_{1}<d_{2}<a_{2}<(T+1) a_{2}<\cdots<d_{k}<a_{k}<$ $(T+1) a_{k}<d_{k+1}$ such that

$$
\begin{gathered}
f(x)<\frac{d_{j}}{D}, \quad x \in\left[0, d_{j}\right], 1 \leq j \leq k+1, \\
f(x)>\frac{a_{h}}{C}, \quad x \in\left[a_{h},(T+1) a_{h}\right], 1 \leq h \leq k .
\end{gathered}
$$

By the assumption, (2.18), we know that the BVP (1.2) has at least $2 k-1$ nonnegative solutions $x_{i}(i=1,2, \ldots, 2 k-1)$ in $\overline{P_{d_{k}}}$. At the same time, it follows from Theorem 2.1 and (2.18) that the BVP (1.2) has at least three nonnegative solutions $u, v$, and $w$ in $\overline{P_{d_{k+1}}}$ 
such that

$$
\begin{gathered}
\|u\|<d_{k}, \quad a_{k}<\min _{t \in[2, T]} v(t), \quad\|w\|>d_{k}, \\
\min _{t \in[2, T]} w(t)<a_{k} .
\end{gathered}
$$

Obviously, $v$ and $w$ are different from $x_{i}(i=1,2, \ldots, 2 k-1)$. Therefore, the BVP (1.2) has at least $2 k+1$ nonnegative solutions in $\overline{P_{d_{k+1}}}$, which shows that this conclusion also holds for $m=k+1$. The proof is complete.

\section{Acknowledgment}

This work was supported by the NSF of Gansu Province of China (3ZS042-B25-020).

\section{References}

[1] R. P. Agarwal, Difference Equations and Inequalities, 2nd ed., Monographs and Textbooks in Pure and Applied Mathematics, vol. 228, Marcel Dekker, New York, 2000.

[2] R. P. Agarwal, M. Bohner, and P. J. Y. Wong, Eigenvalues and eigenfunctions of discrete conjugate boundary value problems, Computers \& Mathematics with Applications 38 (1999), no. 3-4, 159183.

[3] R. P. Agarwal and J. Henderson, Positive solutions and nonlinear eigenvalue problems for thirdorder difference equations, Computers \& Mathematics with Applications 36 (1998), no. 10-12, 347-355.

[4] R. P. Agarwal and P. J. Y. Wong, Advanced Topics in Difference Equations, Mathematics and Its Applications, vol. 404, Kluwer Academic, Dordrecht, 1997.

[5] R. P. Agarwal and F.-H. Wong, Existence of positive solutions for higher order difference equations, Applied Mathematics Letters 10 (1997), no. 5, 67-74.

[6] D. J. Guo and V. Lakshmikantham, Nonlinear Problems in Abstract Cones, Notes and Reports in Mathematics in Science and Engineering, vol. 5, Academic Press, Massachusetts, 1988.

[7] P. Hartman, Difference equations: disconjugacy, principal solutions, Green's functions, complete monotonicity, Transactions of the American Mathematical Society 246 (1978), 1-30.

[8] J. Henderson, Positive solutions for nonlinear difference equations, Nonlinear Studies 4 (1997), no. $1,29-36$.

[9] J. Henderson and P. J. Y. Wong, On multiple solutions of a system of $m$ discrete boundary value problems, Zeitschrift für Angewandte Mathematik und Mechanik 81 (2001), no. 4, 273-279.

[10] M. A. Krasnosel'ski1̌, Positive Solutions of Operator Equations, P. Noordhoff, Groningen, 1964.

[11] R. W. Leggett and L. R. Williams, Multiple positive fixed points of nonlinear operators on ordered Banach spaces, Indiana University Mathematics Journal 28 (1979), no. 4, 673-688.

[12] R.-J. Liang, Y.-H. Zhao, and J.-P. Sun, A new theorem of existence to fourth-order boundary value problem, International Journal of Differential Equations and Applications 7 (2003), no. 3, 257262.

[13] F. Merdivenci, Green's matrices and positive solutions of a discrete boundary value problem, Panamerican Mathematical Journal 5 (1995), no. 1, 25-42.

[14] _ Two positive solutions of a boundary value problem for difference equations, Journal of Difference Equations and Applications 1 (1995), no. 3, 263-270.

[15] J.-P. Sun, Positive solution for BVPs of fourth order difference equations, Indian Journal of Pure and Applied Mathematics 36 (2005), no. 7, 361-370.

[16] J.-P. Sun and W.-T. Li, Multiple positive solutions of a discrete difference system, Applied Mathematics and Computation 143 (2003), no. 2-3, 213-221. 
[17] P. J. Y. Wong, Positive solutions of difference equations with two-point right focal boundary conditions, Journal of Mathematical Analysis and Applications 224 (1998), no. 1, 34-58.

[18] P. J. Y. Wong and R. P. Agarwal, Further results on fixed-sign solutions for a system of higher-order difference equations, Computers \& Mathematics with Applications 42 (2001), no. 3-5, 497-514.

[19] B. Zhang, L. Kong, Y. Sun, and X. Deng, Existence of positive solutions for BVPs of fourth-order difference equations, Applied Mathematics and Computation 131 (2002), no. 2-3, 583-591.

Jian-Ping Sun: Department of Applied Mathematics, Lanzhou University of Technology, Lanzhou, Gansu 730050, China

E-mail address: jpsun@lut.cn 